\title{
FRIENDS OF THE UNIVERSITY OF IOWA LIBRARIES MEMBERSHIP LIST
}

September, 1973

\section{Patrons}

Mr. Maury A. Bromsen, Boston, Massachusetts

Martin H. Burge

Mr. Lawrence O. Cheever, Nixa, Missouri

Mrs. Leslie Douglas, Washington, D. C.

Mr. and Mrs. Owen Elliott, Cedar Rapids, Iowa

Mrs. Lewis C. Frank, Jr., New York, New York

Mr. and Mrs. William Randolph Hearst, Jr., New York, New York

Institute of Hydraulic Research, Iowa City, Iowa

Mr. Karl Kiedaisch, Jr., Forest Hills, New York

Dr. R. N. Larimer, Sioux City, Iowa

Bernice Elizabeth Leary

Thomas O. Mabbott

Mrs. Thomas O. Mabbott, New York, New York

Dr. John Martin, Clarinda, Iowa

Miss Hazel Meek, Washington, Iowa
Mrs. Louise R. Noun, Des Moines, Iowa

Mrs. Gladys W. Richards, Bridgewater, Massachusetts

Mr. Paul C. Richards, Bridgewater, Massachusetts

Mrs. Leo W. Schwarz, New York, New York

Dr. Harold Lincoln Thompson, Los Angeles, California

Mrs. Sara Conn Thompson, Los Angeles, California

The University of Iowa Foundation, Iowa City, Iowa

Mr. Clarence M. Updegraff, San Francisco, California

Mr. Jacob Van Ek, Boulder, Colorado

Mrs. Henry A. Wallace, South Salem, New York

Mr. Henry B. Wallace, West Des Moines, Iowa

Mr. Robert B. Wallace, Doylestown, Pennsylvania

\section{Sustaining}

Mrs. Julia Bartling, Iowa City, Iowa

Mr. and Mrs. Dale M. Bentz, Iowa City, Iowa

Mr. and Mrs. Homer L. Calkin, Arlington, Virginia

Mr. C. David Cornell, Davidson, North Carolina

Mr. Robert G. Davis, Kent, Connecticut

Miss Eve Drewelowe, Boulder, Colorado

Mr. Leslie W. Dunlap, Iowa City, Iowa

Mr. Charles L. Eble, Iowa City, Iowa

Mr. Edwin B. Green, Iowa City, Iowa
Mr. Reeves Hall, Independence, Iowa

Hertzberg-New Method, Inc., Jacksonville, Illinois

Mrs. Frances H. Ingham, Des Moines, Iowa

Dr. and Mrs. Whealen M. Koontz, Cedar Rapids, Iowa

Mr. and Mrs. Wayne Rawley III, Iowa City, Iowa

Mr. John A. Taylor, Washington, Pennsylvania

Mrs. Robert A. Wilcox, Iowa City, Iowa

Mr. M. Dean Zenor, Iowa City, Iowa 


\section{Contributing}

Mr. and Mrs. W. O. Aydelotte, Iowa City, Iowa

Mr. Robert J. Barry, Jr., New Haven, Connecticut

Mr. Ray E. Bazire, Sanibel Island, Florida

Dr. and Mrs. William B. Bean, Iowa City, Iowa

Dr. and Mrs. Michael Bonfiglio, Coralville, Iowa

Mr. and Mrs. Willard L. Boyd, Iowa City, Iowa

Miss Katherine A. Brose, Oakland, California

Miss Lois Davidson, Grinnell, Iowa

Mr. Lowell R. Duhrsen, Las Cruces, New Mexico

Mr. and Mrs. Harry B. Dunlap, Iowa City, Iowa

Cdr. Don Farran USN (Ret), Rowan, Iowa

Mr. and Mrs. John C. Gerber, Iowa City, Iowa

Dr. and Mrs. John S. Greenleaf, Iowa City, Iowa

Mr. and Mrs. Don Guthrie, Iowa City, Iowa

Mr. Frank S. Hanlin, Iowa City, Iowa

Mr. and Mrs. Loren Hickerson, Iowa City, Iowa

Dr. Herbert J. Hodges, Davenport, Iowa

Mr. Delar C. Homan, Lindsborg, Kansas

Mr. and Mrs. H. Clark Houghton, Iowa City, Iowa

Mr. Albert A. Howard, Portland, Maine

Mr. John R. Kleinschmidt, Grinnell, Iowa

Mr. and Mrs. Richard M. Kolbet, Iowa City, Iowa

Dr. Herberta M. Lundegren, State College, Pennsylvania

Mr. Kenneth P. MacDonald, Des Moines, Iowa

Mr. Tom J. McHale, Dallas, Texas

Mr. and Mrs. Enzo O. Macagno, Iowa City, Iowa

Dr. Alfred S. Martin, Philadelphia, Pennsylvania

Mr. Kenneth Nebenzahl, Chicago, Illinois

Mrs. Ellis H. Newsome, Iowa City, Iowa

Mr. Francis J. Paluka, Iowa City, Iowa

Mr. and Mrs. Stow Persons, Iowa City, Iowa

Mrs. Ruby E. Powles, Marion, Iowa

Miss Josephine A. Rocca, Sheboygan, Wisconsin

Mr. and Mrs. Hunter Rouse, Iowa City, Iowa

Mr. and Mrs. David G. Schaal, Iowa City, Iowa

Mr. and Mrs. John E. Simmons, Iowa City, Iowa

Mrs. Robert I. Sinn, East Dubuque, Illinois

Mr. and Mrs. D. C. Spriestersbach, Iowa City, Iowa

Mr. James E. Stronks, Iowa City, Iowa

Mr. J. Richard Wilmeth, Iowa City, Iowa

Miss Grace Wormer, Iowa City, Iowa

Mr. and Mrs. Thomas Yoseloff, Colts Neck, New Jersey

\section{Regular}

Mr. and Mrs. David A. Aamodt, Iowa City, Iowa

Mr. Frank T. Allen, Iowa City, Iowa

Mr. and Mrs. Oren Alt, Iowa City, Iowa
American Antiquarian Society, Worcester, Massachusetts

Mr. Clarence A. Andrews, Houghton, Michigan

Mr. and Mrs. David Archie, Des Moines, Iowa 
Arizona State University Library, Tempe, Arizona

Mr. William G. Asp, Iowa City, Iowa

Auburn University Library, Auburn, Alabama

Miss Vera J. Bacon, Iowa City, Iowa

Miss Frances E. Baker, Poughkeepsie, New York

Miss Sandra Ballasch, Iowa City, Iowa

Mrs. Gladys S. Bartholow, Washington, Iowa

Baylor University Library, Waco, Texas

Mr. Samuel L. Becker, Iowa City, Iowa

Mr. and Mrs. Arthur Benton, Iowa City, lowa

Mr. Dale F. Bentz, Richmond, California

Mr. and Mrs. Thomas E. Bentz, North Oxford, Massachusetts

Miss Rose Mary Berning, Iowa City, Iowa

Mr. and Mrs. Richard J. Bloesch, Iowa City, Iowa

Mr. O M Brack, Jr., Tempe, Arizona

Mr. Larry C. Bruse, Iowa City, Iowa

Mr. Donald C. Bryant, Iowa City, Iowa

Buffalo and Erie County Public Library, Buffalo, New York

Mrs. B. R. Burkhart, Iowa City, Iowa

Ms. Susan Graeber Cagni, San Diego, Califormia

Mrs. Virgil R. Carlson, Kensington, Maryland

Carnegie-Mellon University Library, Pittsburgh, Pennsylvania

Mr. Thomas L. Carney, Cedar Rapids, Iowa

Cedar Rapids Public Library, Cedar Rapids, Iowa

Mr. David R. Cheney, Toledo, Ohio

Ms. Helen S. Clark, Iowa City, Iowa
Mr. Jim E. Cole, Iowa City, Iowa

Mr. Carroll Coleman, Iowa City. Iowa

Mrs. Maria M. Collura, West Liberty, Iowa

Dr. and Mrs. Hunter H. Comly, Iowa City, Iowa

Council Bluffs Public Library. Council Bluffs, Iowa

Dr. and Mrs. Dario A. Covi, Durham, North Carolina

Mr. Robert W. Cryder, Iowa City. Iowa

Mr. William H. Cumberland, Storm Lake, Iowa

Mr. David S. Curry, Iowa City, Iowa

Mr. Charles D. Cuttler, Iowa City. Iowa

Davenport Public Library, Davenport, Iowa

Mr. D. A. Davis, Ottumwa, Iowa

Mr. and Mrs. J. Wayne Deegan. Iowa City, Iowa

Des Moines Public Library, Des Moines, Iowa

Miss Shirley B. Dick, Keokuk, Iowa

Mr. Jack W. Dickey, Iowa City. Iowa

Mr. Richard Dilley, Macomb. Illinois

Mr. W. J. Dixon, Lake Bluff. Illinois

Dr. John M. Dorsey, Highland Park, Michigan

Mr. Rhodes Dunlap, Iowa City. Iowa

East Carolina University Library. Greenville, North Carolina

Miss Eva H. Erickson, Iowa City. Iowa

Mrs. Robert E. Evans, Iowa City. Iowa

Miss Signi Falk, Cedar Rapids. Iowa

Mr. Thomas Farrell, Iowa City, Iowa

Mr. and Mrs. Ronald L. Fingerson, Emporia, Kansas

Mrs. Richard Fisher, Keota, Iowa

Mr. James Fitzmaurice, Flagstaff. Arizona 
Miss Frances M. Flanagan, St. Joseph, Missouri

Miss Frances J. Flick, Tucson, Arizona

Miss Helen E. Focht, Iowa City, Iowa

Mr. Be'la Foltin, Jr., Denton, Texas

Mr. Gene E. Fries, Cedar Rapids, Iowa

Miss Nina A. Frohwein, Iowa City, Iowa

Mrs. J. Curtis Frymoyer, Wilton, Iowa

Mrs. Anneliese M. Funke, Iowa City, Iowa

Mrs. William K. Gaeddert, Iowa City, Iowa

Mrs. Harry F. Garrett, Corydon, Iowa

Mr. Lawrence E. Gelfand, Iowa City, Iowa

Mr. Ralph E. Giesey, Iowa City, Iowa

Mrs. Milton B. Glick, Darien, Connecticut

Miss Anna A. Goldsberry, Iowa City, Iowa

Mr. Jeffrey Goon, New York, New York

Mr. and Mrs. C. Bruce Gould, Hopewell, New Jersey

Mr. William A. Graf, Iowa City, Iowa

Mr. John E. Grant, Iowa City, Iowa

Mr. and Mrs. Richard S. Green, Iowa City, Iowa

Miss Judith K. Groendyke, Iowa City, Iowa

Mr. and Mrs. Charles A. Hale, Iowa City, Iowa

Lathrop C. Harper, Inc., New York, New York

Mr. and Mrs. Peter J. Hartford, Oxford, Iowa

Mr. J. D. Hartzler, Wellman, Iowa

Mrs. E. H. Hatfield, Waterloo, Iowa

Mrs. James Hearst, Cedar Falls, Iowa

Mr. James A. Hedges, Hyattsville, Maryland
Mr. and Mrs. Robert M. Hedges, Hagerstown, Maryland

Miss Judith D. Hendershot, Iowa City, Iowa

Mrs. Forrest Hickman, Iowa City, Iowa

Miss Jane L. Hildebrand, Brunswick, Georgia

Miss Clara Hinton, Iowa City, Iowa

Mr. O. A. Hitchcock, Iowa City, Iowa

Miss Suzanne J. Hosford, Des Moines, Iowa

Mr. and Mrs. Marion L. Huit, Iowa City, Iowa

Mr. and Mrs. John F. Huntley, Iowa City, Iowa

Mr. Michael D. Hurley, Los Angeles, California

Indiana State University Library, Terre Haute, Indiana

Iowa Book and Supply Company, Iowa City, Iowa

Iowa State University Library, Ames, Iowa

Mr. W. R. Irwin, Iowa City, Iowa

Mr. Donald F. Jackson, West Branch, Iowa

Mr. William V. Jackson, Park Ridge, Illinois

Dr. and Mrs. Lewis E. January, Iowa City, Iowa

Miss Rebecca L. Johnson, Iowa City, Iowa

Mr. Howard R. Jones, Solon, Iowa

Mr. Walter Kahoe, Moylan, Pennsylvania

Mr. John C. Kemmerer, New York, New York

Miss Dorothy Kestel, Coralville, Iowa

Miss Margaret N. Keyes, Iowa City, Iowa

Miss Dorothy M. Kibler, Iowa City, Iowa

Mrs. Lester L. Kluever, Atlantic, Iowa

Dr. Paul A. Knights, Meadville, Pennsylvania 
Mrs. James A. Kohler, Iowa City, Iowa

Mrs. John Lagomarcino, Jr., Arlington, Virginia

Dr. and Mrs. Howard B. Latourette, Iowa City, Iowa

Dr. Lillian B. Lawler, Iowa City, Iowa

Miss Hope Leech, Chicago, Illinois

Mr. Roger R. Leech, Chicago, Illinois

Mr. and Mrs. Desmond LeighHunt, Durban, South Africa

Mrs. Hrvoje R. Lorkovic, Iowa City, Iowa

Louisiana State University Library, Baton Rouge, Louisiana

Louisiana State University in New Orleans Library, New Orleans, Louisiana

Miss Ann M. Loventhal, Iowa City, Iowa

Mr. and Mrs. Albert T. Luper, Iowa City, Iowa

Mr. Marion R. McCaulley, Austin, Texas

Mr. Robert A. McCown, Iowa City, Iowa

Mr. and Mrs. Marshall McKusick, Iowa City, Iowa

Miss Esther M. McNutt, Rock Island, Illinois

Mr. Michael Gene Maddigan, Cedar Rapids, Iowa

Miss Lucia A. Marino, Iowa City, Iowa

Mrs. William E. Marks, Iowa City, Iowa

Mr. Karl K. Merker, Iowa City, Iowa

Mr. Robert F. Metzdorf, North Colebrook, Connecticut

Pauline Millen, Books, Des Moines, Iowa

Mr. Dwight M. Miller, West Branch, Iowa

Minnesota Historical Society, St. Paul, Minnesota

Mrs. Pearl J. Minor, Iowa City, Iowa

Mr. Arthur H. Minters, New York, New York
Mr. and Mrs. Albert R. Mitchell. Canyon, Texas

Mr. and Mrs. Leslie G. Moeller, Iowa City, Iowa

Mr. and Mrs. William M. Moldoff, New York, New York

Mrs. Robert W. Munn, Iowa City, Iowa

Mr. and Mrs. Richard L. Murray, Columbia, Maryland

Muscatine Public Library, Muscatine, Iowa

Miss Virginia A. Myers, Iowa City, Iowa

Mr. John J. Newman, Jr., Cedar Rapids, Iowa

Niedersächsische Staats-u Universitäts-Bibliothek, Göttingen, Germany

Miss Mary E. Noble, Iowa City, Iowa

North Texas State University Library, Denton, Texas

Northwestern University Library, Evanston, Illinois

Mr. Ferner Nuhn, Claremont, California

Mr. and Mrs. William G. Nusser, Iowa City, Iowa

Mr. and Mrs. Dean G. Oakes, Iowa City, Iowa

Miss Velva J. Osborn, Iowa City, Iowa

Miss M. Jean Paige, Iowa City, Iowa

Mr. F. D. Paxton, Albuquerque, New Mexico

Miss Jane M. Phillips, Coralville, Iowa

Mr. and Mrs. J. R. Porter, Iowa City, Iowa

Mr. and Mrs. Fred M. Pownall, Iowa City, Iowa

Mr. Keith A. Rageth, Iowa City, Iowa

Mr. Marion E. Rains, Oskaloosa, Iowa

Miss Etta H. Rasmussen, Iowa City, Iowa

Mr. Harry M. Reed, Cedar Falls, Iowa 
Mr. Kenneth W. Rendell, Somerville, Massachusetts

Mr. Earl M. Rogers, Iowa City, Iowa

Mr. Malcolm Rohrbough, Iowa City, Iowa

Mr. and Mrs. William C. Roselle, Thiensville, Wisconsin

Mr. Gordon S. Rowley, Victoria, British Columbia, Canada

Mr. Leland L. Sage, Cedar Falls, Iowa

Mr. and Mrs. Norman Sage, Solon, Iowa

Mr. Gordon W. Searle, Iowa City, Iowa

Mr. Harlan L. Sifford, Iowa City, Iowa

Mrs. Charlotte M. Smith, Newton, Iowa

Southern Illinois University Libraries, Carbondale, Illinois

Stanford University Library, Stanford, California

Mr. Donald H. Stefanson, Holly Springs, Mississippi

Miss Ada M. Stoflet, Iowa City, Iowa

Miss Doris J. Stuck, Iowa City, Iowa

Mr. Curtis W. Stucki, Seattle, Washington

Mr. and Mrs. Dewey B. Stuit, Iowa City, Iowa

Mr. and Mrs. Robert S. Tate, Jr., Iowa City, Iowa

Mr. Thomas T. Thalken, Iowa City, Iowa

Mr. and Mrs. M. S. Thostenson, Iowa City, Iowa

Dr. and Mrs. Franklin H. Top, Iowa City, Iowa

Mr. Robert L. Underbrink, Carlinville, Illinois

University of Arizona Library, Tucson, Arizona

University of California Library, Berkeley, California

University of California Library, San Diego, La Jolla, California
University of California Library, Santa Barbara, California

University of Chicago Library, Chicago, Illinois

University of Illinois Library, Urbana, Illinois

University of Maryland Library, College Park, Maryland

University of Michigan Library, Ann Arbor, Michigan

University of Missouri Library, Columbia, Missouri

University of Northern Iowa Library, Cedar Falls, Iowa

University of Pittsburgh, Graduate School of Library and Information Sciences, Pittsburgh, Pennsylvania

University of South Carolina Library, Columbia, South Carolina

University of Toronto Library, Toronto, Ontario, Canada

University of Washington Libraries, Seattle, Washington

University of Wisconsin-La Crosse Library, La Crosse Wisconsin

University of Wisconsin-Milwaukee Library, Milwaukee, Wisconsin

University of Wyoming Library, Laramie, Wyoming

Mr. Karl H. Van Ausdal, Iowa City, Iowa

Mr. Allan D. Vestal, Iowa City, Iowa

Mr. and Mrs. Carl S. Vestling, Iowa City, Iowa

Miss Carol Sue Vogt, Iowa City, Iowa

Mrs. Kathleen B. Wachel, Iowa City, Iowa

Mr. Norman J. Waffle, Marion, Iowa

Wahlert Memorial Library, Loras College, Dubuque, Iowa

Mr. John J. Walsdorf, New Berlin, Wisconsin

Washington University Libraries, St. Louis, Missouri 
Mr. Stanley Wawzonek, Iowa City, Iowa

Mr. Otha D. Wearin, Hastings, Iowa

Dr. William J. Weiersheuser, Peoria, Illinois

Mr. Theodore N. Weissbuch, Claremont, California

Ms. Hayden E. Wells, New York, New York

Mr. Dennis C. Wendell, Ames, Iowa

Mr. and Mrs. Frederick Wezeman, Iowa City, Iowa

Mrs. Charles A. Wilhite, Iowa City, Iowa
Mr. Melvin Wolfson, Chappaqua, New York

Miss Carla J. Wood, Iowa City, Iowa

Mr. Robert S. Wood, Iowa City, Iowa

Miss Helen Wroe, Keokuk, Iowa

Mr. Emerson G. Wulling, La Crosse, Wisconsin

Miss Helen E. Zeller, Iowa City, Iowa

Mrs. Curt A. Zimansky, Iowa City, Iowa

\section{NOTES ON CONTRIBUTORS}

MILDRED WIRT BENSON is a reporter for the Toledo Times, an aviation columnist for the Blade, and a specialist in the writing of teenage mystery stories. A native of Ladora, Iowa, she received both bachelor's and master's degrees from The University of Iowa.

THOMAS OLLIVE MABBOTT (1898-1968) began his teaching career at Northwestern University, continued it at Hunter College for thirtyodd years, and was visiting professor at the Universities of Chicago, California, Duke and St. John's. He collected Greek and Roman coins and fifteenth-century block prints, he edited works by William Cullen Bryant, Washington Irving, and Walt Whitman, he was co-editor of a volume in the great Columbia edition of Milton, but he is probably best known as the outstanding Poe scholar of his time.

ROBERT A. McCOWN is Manuscripts Librarian at The University of Iowa Libraries. His article on Chautauqua is, in part, the outgrowth of a talk he gave to the Iowa County Federated Women's Club. He is currently doing research toward an article on an Iowa country newspaper editor as well as preparing a study of conservative groups in Iowa since 1945. 